\title{
IL 50 ANNIVERSARIO DEL NOBEL A GIULIO NATTA
}

\author{
$\operatorname{ITALO~PASQUON~(*)}$
}

SuNTO. - L'11 marzo 1954 Giulio Natta sintetizzava il polipropilene isotattico. Si trattava della prima sintesi, in laboratorio, di un polimero avente una struttura ordinata, simile solo a quelle che si trovano in alcuni polimeri naturali quali, ad esempio, la gomma naturale e la cellulosa. Si comprende come, nel suo discorso di prammatica alla cerimonia di consegna del Premio Nobel, il 10 dicembre 1963, il prof. Fredga dell'Accademia Svedese abbia potuto dire che "Il monopolio della natura è stato annullato in seguito alle ricerche del prof. Natta e della sua Scuola". Con la sintesi del polipropilene Natta aveva scoperto la "polimerizzazione stereospecifica", definita nel 1954, da parte di Flory, uno dei massimi cultori della scienza dei polimeri, a sua volta Premio Nobel per la Chimica nel 1974 e da Tobolsky "una rivoluzione" nel campo della chimica macromolecolare. Natta è stato, da allora, universalmente ritenuto il "padre" della polimerizzazione stereospecifica. Nella presentazione del numero di giugno del 1961, a lui dedicato, della rivista statunitense "Journal of Polymer Science" si legge in proposito: "Raramente un contributo scientifico ha sollevato tale profondo e fondamentale interesse ed è stato seguito da tale rapido sviluppo tecnico come la serie di pubblicazioni del professor Natta e dei suoi collaboratori sulla polimerizzazione stereospecifica...”. E ancora: "II professor Natta mantiene un'indiscussa leadership in questo campo della chimica macromolecolare e seguita a stupire i suoi colleghi con continue ed inattese scoperte". La rivoluzione apportata da Natta nel campo della chimica macromolecolare ha coinvolto l'intero mondo scientifico ed industriale specialistico del settore, nel senso che, ben presto, dopo il 1954-1955, buona parte dei laboratori di ricerca universitari ed industriali che nelle varie parti del mondo si occupavano di chimica macromolecolare finì col dedicarsi, in misura più o meno rilevante, ad attività riguardanti la neonata polimerizzazione stereospecifica. I risultati di queste ricerche sono contenuti in circa un migliaio di pubblicazioni scientifiche e alcune centinaia di brevetti usciti dall'allora Istituto di Chimica Industriale del Politecnico di Milano tra il 1955 e il 1971 (concentrate tra gli anni 1955 e 1967). L'importanza sul piano commerciale del polipropilene si evince dal fatto che la produzione, a livello mondiale, delle varie tipologie di polipropileni ammonta oggi

(*) Istituto Lombardo Accademia di Scienze e Lettere, Politecnico di Milano, Italia. E-mail: ropasquon@gmail.com 
a circa 60 milioni di tonnellate/anno. Si stima che il loro valore economico, sempre a livello mondiale, si avvicini, tra tutti i prodotti di sintesi che hanno un nome, al secondo posto, dopo i polietileni, assieme all'ammoniaca, e prima di altri prodotti quali i polimeri dello stirene, del cloruro di vinile, i nylon, ecc. Dal canto loro il polibutadiene 1,4-cis e i copolimeri a base di etilene-propilene occupano oggi, a livello mondiale, in termini di produzione e valore commerciale, rispettivamente il secondo e terzo posto tra gli elastomeri sintetici, dopo le gomme stirene-butadiene (SBR), preparate già prima della seconda guerra mondiale. Chi fosse interessato ad una più completa conoscenza della personalità e delle attività scientifiche e didattiche di Giulio Natta, dei suoi rapporti con il mondo universitario e industriale a livello mondiale, può consultare il sito internet www.giulionatta.it che contiene l'"Archivio di Giulio Natta" (oltre 40.000 pagine).

$* * *$

AвSTRACT. - On March 11, 1954 Giulio Natta synthetized isotactic polypropylene. It was the first synthesis, in laboratory, of a polymer having a sterically ordered structure, similar to those found in some natural polymers like, for instance, natural rubber and cellulose. This meaningful aspect was emphasized by Prof. Fredga of the Swedish Academy in his speech at the customary ceremony of Nobel Prize on December 10, 1963, when he stated "Natta has broken the monopoly of nature". With the polypropylene synthesis Natta discovered the "stereospecific polymerization" defined by Toblosky (Princeton University) and by Flory, one of the most eminent scientist in the macromolecular chemistry and future Nobel laureate in Chemistry (1974), a "revolution" in the macromolecular field. Natta was recognized as the "father" of stereospecific polymerization. The Journal of Polymer Science, vol. 51 (1961), dedicated to Natta, reports "Seldom a scientific contribution aroused such a deep and fundamental interest and was followed by such a rapid technical development as the series of publications by Professor Natta and his coworkers on the stereospecific polymerization. Yet Professor Natta has succeeded in maintaining undisputed leadership in this field of polymer chemistry and continues to surprise his colleagues with new and unexpected results". The revolution brought by Natta in the field of macromolecular chemistry involved the entire scientific and industrial world specialized in that area. Soon after 1954-55 a lot of universities and industrial laboratories around the world started to work on activities concerning the novel stereospecific polymerization. The results of these studies are reported in about a thousand scientific publications and some hundreds of patents issued from the Milan Polytechnic Institute of Industrial Chemistry between 1955 and 1977 (concentrated in 1955-57). The importance of polypropylene from the commercial point of view is evident from the fact that the world production of various types of polypropylene now stands at about 60 million tons/year. It is estimated that the related global economic value approaches the second place over all synthetic products, after polyethylene, together with ammonia, and before other products such as urea, polymers of styrene and vinyl chloride, nylons, etc. For their part 1,4-cis polybutadiene and copolymers based on ethylene and propylene occupy respectively the second and the third place in terms of worldwide production and market value among synthetic elastomers, after styrene-butadiene rubbers (SBR). More information concerning the personality and the scientific and didactic activities of Giulio Natta and his relations with the scientific and industrial world are reported on the internet site www.giulionatta.it containing the "Giulio Natta Archive" (more than 40.000 pages). 
All'inizio degli anni '50, sulla scia di quanto già verificatosi negli USA, si è aperta in Italia l'era della petrolchimica, che attraverso i processi di cracking dei prodotti petroliferi, ha messo a disposizione grandi quantità di prodotti di base, quali l'etilene, il propilene, il butadiene e il benzene.

$\mathrm{Ha}$ avuto così inizio in Italia e in Europa la realizzazione di grandi impianti per la preparazione di un'ampia gamma di prodotti finiti, i più importanti dei quali sono le materie plastiche, le gomme e le fibre sintetiche. E' in questi settori che si collocano le più note ed importanti ricerche di Natta che gli hanno valso il Premio Nobel per la Chimica nel 1963.

Queste ricerche erano iniziate nel 1954 con la sintesi, l'11 marzo di quell'anno, del polipropilene isotattico. Si trattava della prima sintesi, in laboratorio, di un polimero avente una struttura ordinata, simile solo a quelle che si trovano in alcuni polimeri naturali quali, ad esempio, la gomma naturale e la cellulosa.

Si comprende come, nel suo discorso di prammatica alla cerimonia di consegna del Premio Nobel, il 10 dicembre 1963, il prof. Fredga dell'Accademia Svedese abbia potuto dire che "Il monopolio della natura è stato annullato in seguito alle ricerche del prof. Natta e della sua Scuola".

Con la sintesi del polipropilene isotattico, Natta aveva scoperto la "polimerizzazione stereospecifica", definita nel 1954, da parte di Flory, uno dei massimi cultori della scienza dei polimeri, a sua volta Premio Nobel per la Chimica nel 1974 e da Tobolsky "una rivoluzione" nel campo della chimica macromolecolare.

Natta è stato, da allora, universalmente ritenuto il "padre" della polimerizzazione stereospecifica.

Nella presentazione del numero di giugno del 1961, a lui dedicato, della rivista statunitense "Journal of Polymer Science" si legge in proposito: "Raramente un contributo scientifico ha sollevato tale profondo e fondamentale interesse ed è stato seguito da tale rapido sviluppo tecnico come la serie di pubblicazioni del professor Natta e dei suoi collaboratori sulla polimerizzazione stereospecifica...".

E ancora: "II professor Natta mantiene un'indiscussa leadership in questo campo della chimica macromolecolare e seguita a stupire $i$ suoi colleghi con continue ed inattese scoperte".

Analoghi apprezzamenti sono stati fatti da Sir Robinson, Premio Nobel per la Chimica 1954.

Può essere utile rammentare le caratteristiche fondamentali della polimerizzazione stereospecifica, mediante la quale sono ottenibili polimeri stereoregolari (Figs. 1 e 2). 


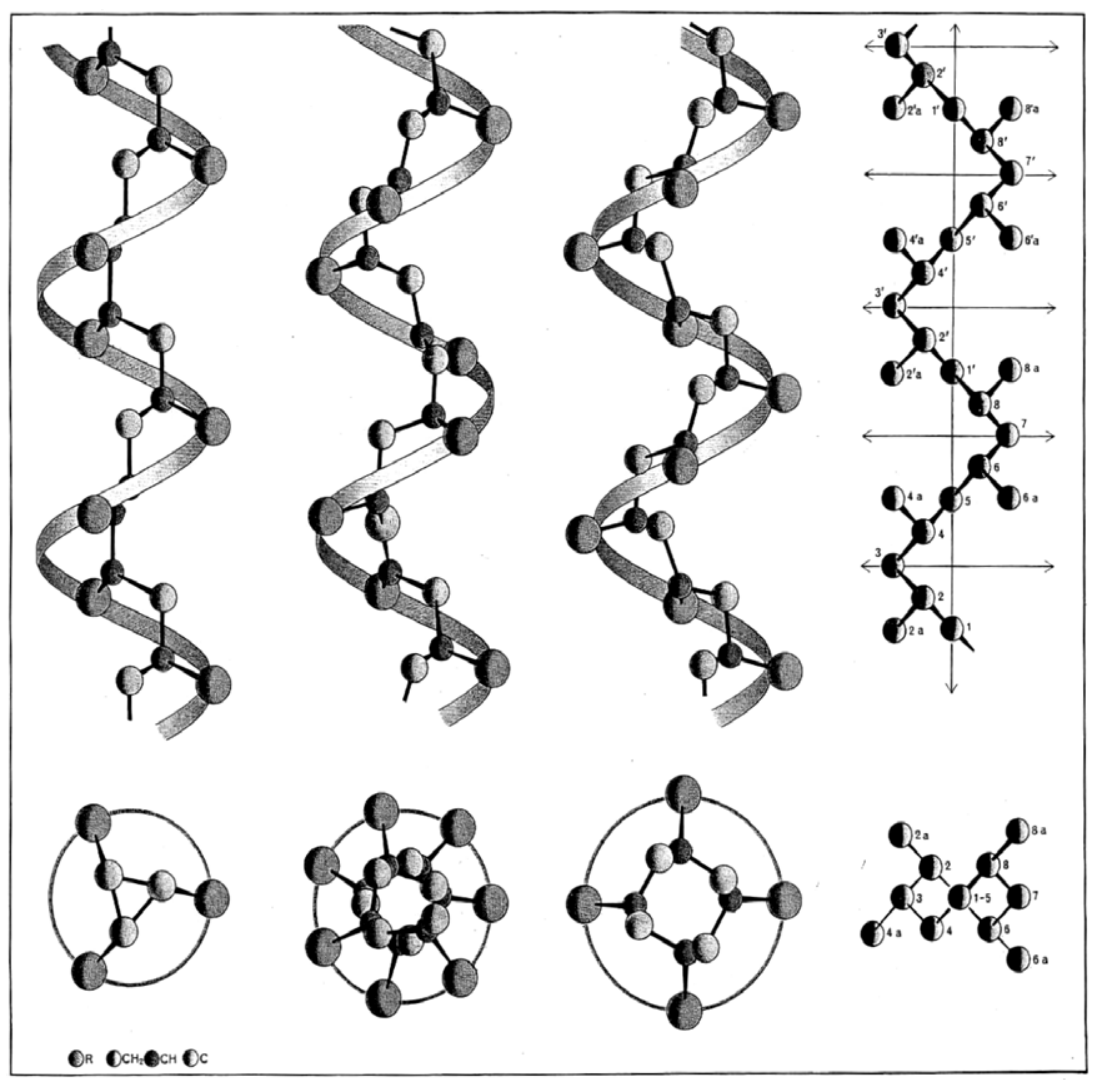

Fig. 1. Rappresentazione schematica di catene polimeriche: a sinistra isotaticche derivanti da monomeri vinilici $\mathrm{CH}_{2}=\mathrm{CHR}$; a destra del polipropilene sindiotattico.

Come noto, le macromolecole che costituiscono i polimeri naturali o sintetici contengono unità monometriche tra loro uguali, unite da legami chimici. Prima della scoperta di Natta, nei polimeri sintetici ottenuti da monomeri di tipo vinilico, la successione delle unità monometriche lungo le catene polimeriche era di tipo disordinato, in particolare dal punto di vista sterico.

La polimerizzazione stereospecifica consentiva, per la prima volta, la sintesi di polimeri ordinati sia chimicamente che stericamente - e quindi cristallizzabili - a partire da monomeri di tipo vinilico o comunque insaturi. 


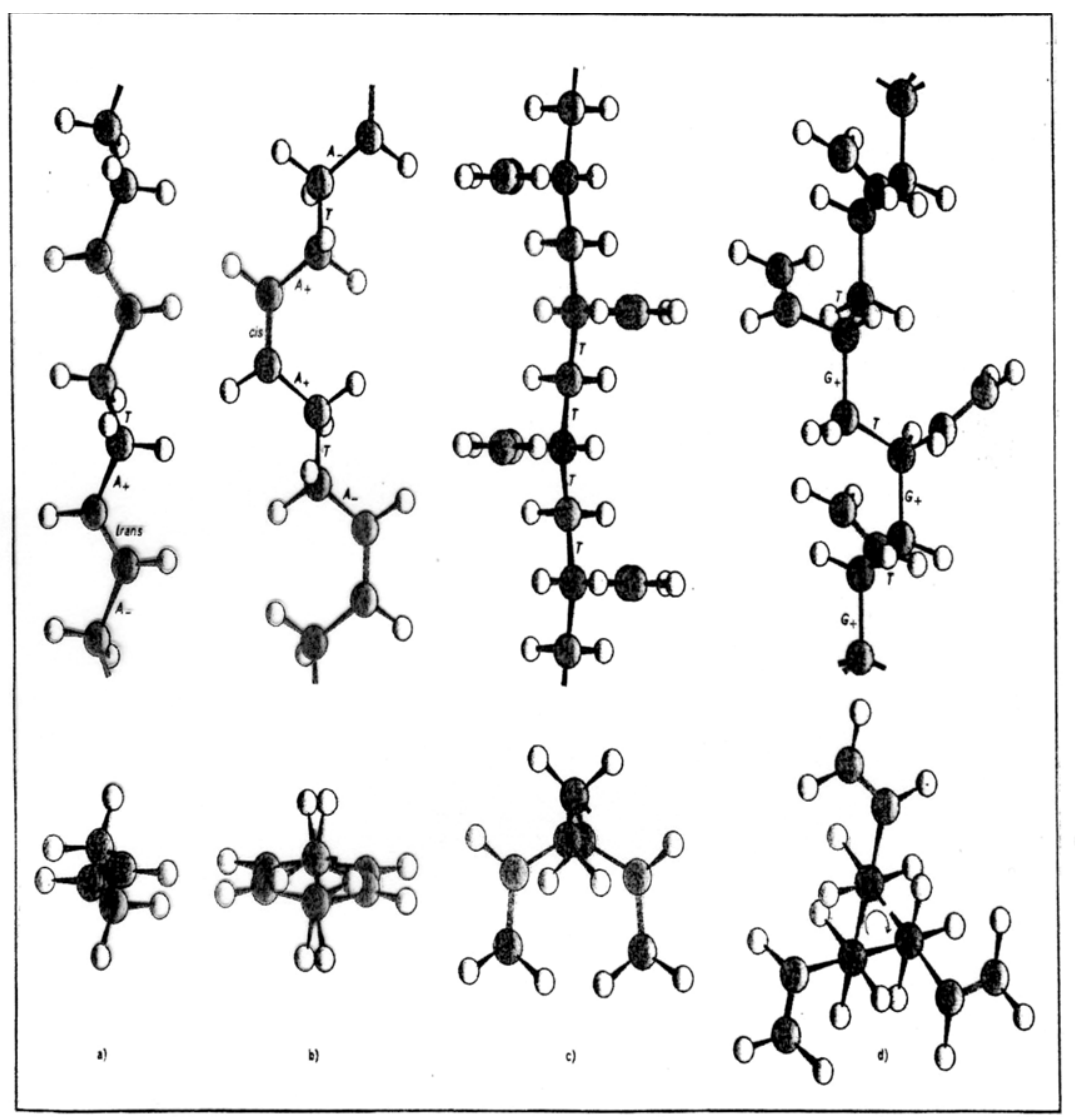

Catene dei polimeri del butadiene allo stato cristallino: a) 1,4-trans; b) 1,4-cis; c) 1,2-sindiotattico; d) 1,2-isotattico (da Pasquon, 1970).

Fig. 2. Catene dei polimeri del butadiene.

In precedenza polimeri di questo tipo si trovavano soltanto in natura (ad es. gomma naturale e guttaperca).

Il polipropilene stereoregolare definito da Natta "isotattico" è una polvere cristallina di densità inferiore a quella dell'acqua, che fonde oltre i $170^{\circ} \mathrm{C}$ e dalla quale sono ottenibili manufatti aventi un carico di rottura di $3-4 \mathrm{~kg} / \mathrm{mmq}$; il polipropilene non stereoregolare (atattico) è una sostanza oleosa.

L'importanza di queste ricerche sul piano più propriamente scientifico non si limita al fatto che la polimerizzazione stereospecifica 
consentiva per la prima volta la sintesi di polimeri stereoregolari ottenuti a partire da monomeri di varia natura. Fondamentali sono stati anche i lavori sulla scoperta di vari sistemi catalitici e sul loro comportamento e quelli sulla determinazione della struttura di sostanze polimeriche, sulle relazioni tra proprietà e struttura e sulle sintesi asimmetriche (Tab. 1 e 2): con questo tipo di sintesi veniva infatti stabilito un legame tra una classe di fenomeni che si verificano in natura e reazioni realizzabili per la prima volta in laboratorio.

La rivoluzione apportata da Natta nel campo della chimica macromolecolare ha coinvolto l'intero mondo scientifico ed industriale specialistico del settore, nel senso che, ben presto, dopo il 1954-1955, buona parte dei laboratori di ricerca universitari ed industriali che nelle varie parti del mondo si occupavano di chimica macromolecolare finì col dedicarsi, in misura più o meno rilevante, ad attività riguardanti la neonata polimerizzazione stereospecifica.

Tab. 1. Temi di ricerca affrontati da Natta e dalla sua Scuola nel campo della polimerizzazione stereospecifica.

Sintesi, caratterizzazione della struttura allo stato cristallino e determinazioni di talune proprietà chimico-fisiche, meccaniche e fisiche di oltre 130 di nuovi tipi di polimeri;

Preparazione, studi e caratterizzazione di sistemi catalitici costituiti da un composto di metallo di transizione e da un composto metallorganico (catalizzatore Ziegler-Natta), oppure da soli composti metallorganici;

Studi sui meccanismi e sulla cinetica di diversi processi di polimerizzazione;

Sintesi di polimeri politattici;

Sintesi asimmetriche;

Sintesi di copolimeri poliolefinici a distribuzione statistica e loro applicazione per la preparazione di elastomeri;

Sintesi di copolimeri cristallini alternanti;

Preparazione e caratterizzazione di elastomeri saturi ed insaturi e di fibre;

Polimeri ad innesto;

Polimeri stereoregolari ottenuti da composti di inclusione;

Impiego di polimeri in campo farmaceutico. 
E per diversi anni questi laboratori si sono essenzialmente ispirati ai lavori di Natta e della sua Scuola, facendo cioè, per lo più, una ricerca di rincorsa.

Non è fuori luogo affermare che la maggior parte dei risultati di rilievo ottenuti nel campo della polimerizzazione stereospecifica in senso lato ha continuato, per diversi anni, a partire dal 1954, a provenire dai laboratori di Natta. Tra il 1954 e la fine degli anni '60 le ricerche sviluppate al Politecnico di Milano hanno portato alla scoperta di oltre 130 nuovi polimeri.

I risultati di queste ricerche sono contenuti in circa un migliaio di pubblicazioni scientifiche e alcune centinaia di brevetti usciti dall'allora Istituto di Chimica Industriale del Politecnico di Milano tra il 1955 e il 1971 (concentrate tra gli anni 1955 e 1967).

Da allora, la polimerizzazione stereospecifica continua ad essere oggetto di studi.

Questo per quanto attiene all'importanza scientifica dei lavori di Natta e della sua Scuola.

\section{Tab. 2. Nuovi polimeri preparati dalla Scuola di Natta.}

\begin{tabular}{|c|c|}
\hline 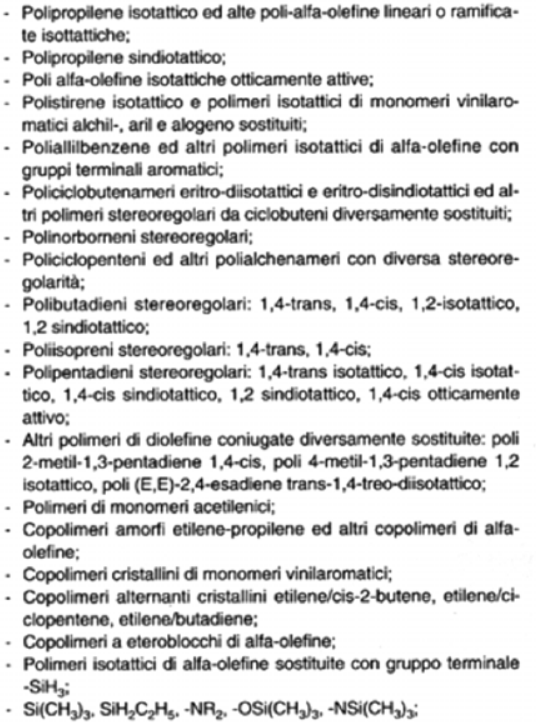 & 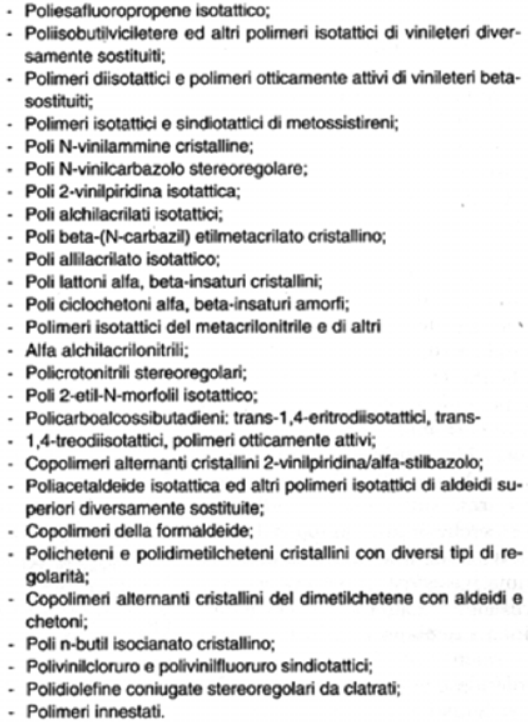 \\
\hline
\end{tabular}


Sul piano applicativo tali ricerche hanno condotto alla scoperta di nuovi tipi di polimeri di rilevante interesse industriale,oltre al polipropilene isotattico (utilizzato nella produzione di materie plastiche aventi svariati impieghi nei settori automobilistico, elettrodomestici, prodotti per la casa, edilizia, tempo libero, imballaggi rigidi e flessibili, fibre sintetiche), quali i copolimeri a base di etilene e propilene e il polibutadiene 1,4-cis, due importanti elastomeri sintetici. Da rilevare che prima di allora il propilene aveva limitate applicazioni, a fronte di quantitativi disponibili relativamente elevati.

Per evidenziare l'importanza a livello applicativo, di queste scoperte può essere ricordato quanto riportato nel numero di novembre 1963 de "La Chimica e l'Industria", nell'editoriale dedicato a Natta, a seguito dell'assegnazione del premio Nobel: “... il campo della chimica industriale è stato 'arato' talmente a fondo che difficilmente ci potrà riservare la sorpresa di scoperte d'importanza altrettanto rilevante".

A oltre 60 anni dalla scoperta fatta da Giulio Natta, questa affermazione non è ancora stata smentita.

Un'analisi approfondita del settore porta anzi a concludere che la scoperta del polipropilene isotattico e della polimerizzazione stereospecifica è stata l'ultima delle grandi scoperte - sul piano applicativo ancora possibili nel campo della chimica industriale tradizionale.

L'importanza sul piano commerciale del polipropilene si evince dal fatto che la produzione, a livello mondiale, delle varie tipologie di polipropileni, che nel 2001 ammontava a 31,6 milioni di tonnellate/anno ammonta oggi a oltre 60 milioni di tonnellate. Si stima che il loro valore economico, sempre a livello mondiale, si avvicini, tra tutti i prodotti di sintesi che hanno un nome, al secondo posto, dopo i polietileni, assieme all'ammoniaca, e prima di altri prodotti quali i polimeri dello stirene, del cloruro di vinile, i nylon, ecc.

Dal canto loro il polibutadiene 1,4-cis e i copolimeri a base di etilene-propilene occupano oggi, a livello mondiale, in termini di produzione e valore commerciale, rispettivamente il secondo e terzo posto tra gli elastomeri sintetici, dopo le gomme stirene-butadiene (SBR), preparate già prima della seconda guerra mondiale.

Questi risultati sono stati coperti da 280 "famiglie" di brevetti industriali e da oltre 4000 brevetti concessi in vari paesi.

$\mathrm{Ci}$ si può ora chiedere in quale modo risultati tanto significativi si siano potuti ottenere in così poco tempo.

E' stato fondamentale l'apporto della Montecatini che ha fornito mezzi economici e che, soprattutto, ha messo a disposizione, nel- 
l'arco di una quindicina d'anni, alcune decine di validi ricercatori (chimici ed ingegneri) per la maggior parte molto giovani, i quali, assieme agli assistenti di Natta (poche unità e anch'essi in maggioranza molto giovani), hanno poi costituito quella che ancor oggi è nota come la "Scuola di Natta".

Ma era essenziale una "mente", con adeguato bagaglio scientifico, in grado di fornire idee e di coordinare un lavoro interdisciplinare che riguardava vari settori della chimica organica, inorganica, metallurgica e macromolecolare, della chimica-fisica, dalla termodinamica alla catalisi, alla cinetica e alla strutturistica, dall'analisi sperimentale (RX, Raman, NMR, IR, UV, ESR, radiochimica), della fisica e dell'ingegneria, fino allo studio delle proprietà fisiche e meccaniche di materie plastiche, elastomeri e fibre.

Natta aveva questi requisiti.

A differenza della maggior parte degli altri Premi Nobel per la chimica, non era uno "specialista". Prima di affrontare il tema della polimerizzazione stereospecifica, Natta aveva svolto ricerche in diversi campi della chimica e della chimico-fisica, con un occhio sempre attento alle applicazioni industriali (vedi Appendice). Il suo primo brevetto è del 1927 e alcuni impianti industriali, frutto delle sue ricerche, sono stati realizzati in Italia e nel mondo.

Un altro merito da riconoscere a Natta è quello di essere stato un precursore, in quanto il rapporto allora instaurato con la Montecatini costituisce il primo caso di collaborazione tra università e industria di ampie dimensioni.

Natta era un lavoratore instancabile. Dopo un'intera giornata trascorsa al Politecnico, nell'Istituto di Chimica industriale - ove, finché lo stato di salute glielo consentì, continuò a tenere le sue lezioni ed a esaminare personalmente ogni allievo - quasi ogni sera, dopo cena, Natta riceveva a turno i suoi collaboratori a casa sua, in via Mario Pagano 54, per discutere di ricerca. Lo stesso si ripeteva nei fine settimana. Alla domenica si pranzava con lui e si riprendevano poi le discussioni. Durante i periodi di vacanza e in qualche week-end alcuni di noi erano ospiti nelle sue case di villeggiatura: alla mattina si passeggiava nei boschi, o si cercavano funghi e al pomeriggio si lavorava. Natta era un profondo conoscitore di funghi, di botanica e di minerali.

Nel contempo Natta orientava anche ricerche svolte presso i vari settori della Montecatini e in alcuni Istituti universitari e del CNR e manteneva stretti rapporti con il mondo esterno, documentati da un ponderoso carteggio che copre essenzialmente l'arco temporale 1950- 
1970, conservato al Politecnico di Milano e riprodotto sul sito internet Archivio di Giulio Natta.

Il materiale conservato concerne le relazioni intercorse con:

- oltre 500 tra personalità scientifiche italiane e straniere (USA, D, GB, F, B, URSS, CH, NL, J, S, CDN, ecc), tra cui alcuni Premi Nobel, e Università e altre istituzioni scientifiche (rapporti non solo epistolari, ma anche scambi di visite e inviti famigliari);

- oltre 300 grandi aziende mondiali (di cui circa la metà statunitensi), per discutere di ricerche ed anche di concessioni di licenze;

- praticamente tutti i settori Montecatini, Montecatini-Edison (Settore Idrocarburi, Settore Resine, Istituto Donegani, ACNA, Settore Progetti e Studi, Settore Prodotti Chimici per l'Industria) nei quali venivano anche effettuati studi e ricerche da lui stesso indirizzati e seguiti;

- l'Amministratore Delegato ing. Piero Giustiniani di Montecatini, per un'ampia gamma di argomenti;

- le società di rappresentanza Montecatini all'estero;

- l'Ufficio Brevetti Montecatini, con il quale seguiva il deposito dei brevetti in Italia e la loro estensione e concessione all'estero, talvolta molto laboriosa e complessa: basti ricordare che il carteggio riguardante la concessione negli USA del brevetto sul polipropilene isotattico (depositato negli USA nel 1954 e concesso solo nel 1963) occupa tre voluminosi raccoglitori;

- il CNR, presso il quale vennero creati il Centro Nazionale di Chimica delle Macromolecole - con diverse sedi in Italia - e l'Istituto Nazionale di Chimica delle Macromolecole (ICM) del cui Consiglio Scientifico Natta venne nominato presidente;

- l'Accademia Nazionale dei Lincei ed altre Accademie e Associazioni scientifico- culturali, italiane e straniere, delle quali era membro;

- il Rettorato del Politecnico e il Preside della Facoltà di Ingegneria; varie riviste e case editrici italiane e straniere.

Natta era un uomo riservato. Sapeva mantenere con tutti rapporti sinceramente umani, anche se mascherati da un apparente distacco, certo dovuto a timidezza. Incuteva rispetto, senza mai alzare la voce. Per tutti era il "Professore".

Egli passo gli ultimi anni della sua vita a Bergamo, accanto alla figlia Franca, ove si spense il 2 maggio 1979, per l'acuirsi della malattia che lo affliggeva, dopo molti anni di sofferenza, sopportata con grande forza d'animo.

Era nato ad Imperia il 26 febbraio 1903. 


\section{APPENDICE}

Principali settori dell' attività scientifica e collaboratori di GIULIO NATTA

Stabilità di soluzioni e applicazione dei raggi $\mathrm{X}$ allo studio di sostanze allo stato cristallino $\mathrm{e}$ di leghe (1923-1936)

\begin{tabular}{|c|c|c|}
\hline Sostanze studiate & Collaboratori & Periodo \\
\hline Cloriti alcalini & G.R. Levi & (1923) \\
\hline Solidi inorganici vari, complessi, spinelli & $\begin{array}{l}\text { M. Strada } \\
\text { L. Passerini } \\
\text { M. Baccaredda }\end{array}$ & $\begin{array}{l}(1928-1932) \\
(1928-1936) \\
(1932-1936)\end{array}$ \\
\hline Leghe, soluzioni solide e casi di isomorfismo & $\begin{array}{l}\text { M. Freri } \\
\text { L. Passerini } \\
\text { R. Pirani }\end{array}$ & $\begin{array}{r}(1927-1928) \\
(1928-1929) \\
(1932)\end{array}$ \\
\hline Sostanze organiche & & \\
\hline Gas e liquidi allo stato solido & $\begin{array}{l}\text { G. Nasini } \\
\text { E. Casazza } \\
\text { R. Rigamonti }\end{array}$ & $\begin{array}{l}(1930) \\
(1930) \\
(1936)\end{array}$ \\
\hline
\end{tabular}

Applicazioni della diffrazione di elettroni allo studio di strutture (1935-1937)

\begin{tabular}{|c|c|c|}
\hline Sostanze studiate & Collaboratori & Periodo \\
\hline Polimeri ed elastomeri vari & R. Rigamonti & (1936) \\
\hline Colloidi & & \\
\hline Sostanze organiche varie & $\begin{array}{l}\text { M. Baccaredda } \\
\text { R. Rigamonti }\end{array}$ & $\begin{array}{l}(1935) \\
(1935)\end{array}$ \\
\hline Cellulosa & M. Baccaredda & (1936) \\
\hline Metalli, leghe e soluzioni solide metalliche & A. Giuriani & (1937) \\
\hline
\end{tabular}


Metanolo e alcooli superiori da CO e $\mathbf{H}_{2}$ (1928-1957)

\begin{tabular}{|c|c|c|}
\hline Argomento & Collaboratori & Periodo \\
\hline $\begin{array}{l}\text { Preparazione e studio di catalizzatori } \\
\text { per la sintesi del metanolo }\end{array}$ & E. Casazza & (1931) \\
\hline $\begin{array}{l}\text { Preparazione e studio di catalizzatori per la } \\
\text { sintesi di alcooli superiori }\end{array}$ & $\begin{array}{l}\text { M. Strada } \\
\text { R. Rigamonti }\end{array}$ & $\begin{array}{r}(1930-1931) \\
(1932)\end{array}$ \\
\hline Studi cinetici sulla sintesi del metanolo & G. Pastonesi & $(1937-1938)$ \\
\hline Misure di absorbimento su catalizzatori & N. Agliardi & (1947) \\
\hline Cinetica della sintesi del metanolo & $\begin{array}{l}\text { I. Pasquon } \\
\text { G. Mazzanti } \\
\text { P. Pino }\end{array}$ & $\begin{array}{r}(1953-1960) \\
(1953-1955) \\
(1953)\end{array}$ \\
\hline Studi su catalizzatori & P. Corradini & (1954) \\
\hline $\begin{array}{l}\text { Studi su catalizzatori per le sintesi } \\
\text { del metanolo a bassa pressione }\end{array}$ & $\begin{array}{l}\text { G. Mazzanti } \\
\text { I. Pasquon }\end{array}$ & $\begin{array}{l}(1955) \\
(1955)\end{array}$ \\
\hline Sintesi di alcooli superiori & I. Pasquon & (1957) \\
\hline
\end{tabular}

Sintesi e polimerizzazione della formaldeide (1932-1933)

\begin{tabular}{|c|c|c|}
\hline Argomento & Collaboratori & Periodo \\
\hline Formaldeide da metanolo & $\begin{array}{l}\text { M. Strada } \\
\text { M. Baccaredda }\end{array}$ & $\begin{array}{l}(1932) \\
(1932)\end{array}$ \\
\hline Polimeri della formaldeide & M. Baccaredda & $(1932-1933)$ \\
\hline
\end{tabular}

Gassificazione del carbone (1932-1938)

\begin{tabular}{|c|c|c|}
\hline Argomento & Collaboratori & Periodo \\
\hline Gassificazione con ossigeno & $\begin{array}{l}\text { M. Strada } \\
\text { R. Rigamonti }\end{array}$ & $\begin{array}{l}(1932) \\
(1938)\end{array}$ \\
\hline
\end{tabular}


Sintesi dell'isottano (1938-1943)

\begin{tabular}{|c|c|c|}
\hline Argomento & Collaboratori & Periodo \\
\hline Sintesi dal gas d'acqua & M. Baccaredda & (1938) \\
\hline Sintesi da isobutilene & M. Baccaredda & $(1939-1943)$ \\
\hline
\end{tabular}

Idrogenazione del furfurolo e di carboidrati (1939-1951)

\begin{tabular}{|c|c|c|}
\hline Argomento & Collaboratori & Periodo \\
\hline Idrogenazione del furfurolo & $\begin{array}{l}\text { R. Rigamonti } \\
\text { E. Beati }\end{array}$ & $\begin{array}{l}(1939-1941) \\
(1939-1941)\end{array}$ \\
\hline Idrogenazione di carboidrati & $\begin{array}{l}\text { R. Rigamonti } \\
\text { E. Beati }\end{array}$ & $\begin{array}{l}(1942-1943) \\
(1942-1943)\end{array}$ \\
\hline Cinetica idrogenazione del glucosio & E. Beati & (1943) \\
\hline Estrazione del furfurolo & E. Beati & (1951) \\
\hline
\end{tabular}

Absorbimento frazionato e sua applicazione al frazionamento di miscele di idrocarburi $\mathrm{C}_{4}$ - Sintesi del butadiene (1942-1953)

\begin{tabular}{|c|c|c|}
\hline Argomento & Collaboratori & Periodo \\
\hline $\begin{array}{l}\text { Messa a punto e studio } \\
\text { dell'absorbimento frazionato }\end{array}$ & G.F. Mattei & (1942) \\
\hline Butadiene da etanolo & R. Rigamonti & (1947) \\
\hline Purificazione del butadiene & R. Rigamonti & (1947) \\
\hline Teoria dell'absorbimento frazionato & $\begin{array}{l}\text { G. Negri } \\
\text { P.L. Gadina }\end{array}$ & $\begin{array}{r}(1952-1953) \\
(1953)\end{array}$ \\
\hline
\end{tabular}

Reazioni successive (1945-1952)

\begin{tabular}{|c|c|c|}
\hline Argomento & Collaboratori & Periodo \\
\hline $\begin{array}{l}\text { Impostazione dello studio cinetico } \\
\text { e verifiche sperimentali }\end{array}$ & M. Simonetta & (1945) \\
\hline Definizione delle equazioni generali & E. Mantica & $(1951-1952)$ \\
\hline
\end{tabular}


Applicazione degli ultrasuoni (1948-1954)

\begin{tabular}{llll}
\hline Argomento & Collaboratori & & Periodo \\
\cline { 1 - 2 } $\begin{array}{l}\text { Studio di alti polimeri e di soluzioni } \\
\text { polimeriche }\end{array}$ & M. Baccaredda & & \\
\cline { 1 - 2 } & & & \\
\hline Studio di liquidi e di miscele liquide & F. Danusso & & $(1948-1954)$ \\
\hline
\end{tabular}

Oxosintesi e reazioni similari (1945-1966)

\begin{tabular}{|c|c|c|}
\hline Argomento & Collaboratori & Periodo \\
\hline $\begin{array}{l}\text { Reazioni con olefine, } \mathrm{CO} \text { e } \mathrm{H}_{2} \text {; studi cinetici; } \\
\text { meccanismi }\end{array}$ & E. Beati & $(1945-1950)$ \\
\hline $\begin{array}{l}\text { Reazioni con olefine, } \mathrm{CO} \text { e } \mathrm{H}_{2} \text {; studi cinetici; } \\
\text { meccanismi }\end{array}$ & $\begin{array}{l}\text { P. Pino } \\
\text { R. Ercoli } \\
\text { S. Castellano } \\
\text { F.G. Barbieri }\end{array}$ & $\begin{array}{r}(1949-1952) \\
(1952-1955) \\
(1954-1955) \\
(1954)\end{array}$ \\
\hline Reazioni con olefine, $\mathrm{CO}$ ed alcooli & $\begin{array}{l}\text { P. Pino } \\
\text { E. Ercoli } \\
\text { E. Mantica }\end{array}$ & $\begin{array}{r}(1950-1952) \\
(1951-1960) \\
(1950)\end{array}$ \\
\hline Reazioni con acetilene, $\mathrm{CO}$, alcooli o acqua & $\begin{array}{l}\text { P. Pino } \\
\text { G.C. Albanesi } \\
\text { I. Pasquon }\end{array}$ & $\begin{array}{r}(1949-1952) \\
(1956-1966) \\
(1961)\end{array}$ \\
\hline
\end{tabular}

Polimerizzazione radicalica di monomeri vinilici (1952-1956)

\begin{tabular}{|c|c|c|}
\hline Argomento & Collaboratori & Periodo \\
\hline $\begin{array}{l}\text { Studio di processi di polimerizzazione del } \\
\text { cloruro di vinile in massa e in sospensione. } \\
\text { Caratterizzazione dei prodotti polimerici }\end{array}$ & $\begin{array}{l}\text { F. Danusso } \\
\text { G. Moraglio } \\
\text { D. Sianesi }\end{array}$ & $\begin{array}{l}(1952-1956) \\
(1952-1954) \\
(1953-1955)\end{array}$ \\
\hline $\begin{array}{l}\text { Polimerizzazione ed oligomerizzazione di } \\
\text { monomeri acrilici e metacrilici ad alta } \\
\text { temperatura }\end{array}$ & F. Danusso & $(1953-1955)$ \\
\hline
\end{tabular}


Complessi carbonilici (1953-1967)

\begin{tabular}{|c|c|c|}
\hline Argomento & Collaboratori & Periodo \\
\hline Complessi di Co: sintesi e struttura & $\begin{array}{l}\text { E. Ercoli } \\
\text { P. Corradini } \\
\text { P. Chini } \\
\text { F. Calderazzo } \\
\text { M. Peraldo } \\
\text { M. Massi Mauri } \\
\text { G. Allegra }\end{array}$ & $\begin{array}{r}(1958-1966) \\
(1953-1959) \\
(1958-1960) \\
(1967) \\
(1961) \\
(1959) \\
(1966)\end{array}$ \\
\hline Complessi di Cr: sintesi e struttura & $\begin{array}{l}\text { R. Ercoli } \\
\text { F. Calderazzo } \\
\text { E. Bernardi } \\
\text { P. Corradini } \\
\text { G. Allegra } \\
\text { E. Mantica } \\
\text { E. Mostardini } \\
\text { G. Pajaro }\end{array}$ & $\begin{array}{r}(1957-1967) \\
(1957-1967) \\
(1959) \\
(1959) \\
(1959) \\
(1959) \\
(1960) \\
(1960)\end{array}$ \\
\hline Complessi di V: sintesi e struttura & $\begin{array}{l}\text { E. Ercoli } \\
\text { F. Calderazzo } \\
\text { P. Corradini } \\
\text { G. Allegra } \\
\text { A. Alberola } \\
\text { R. Cini }\end{array}$ & $\begin{array}{r}(1959-1962) \\
(1959-1963) \\
(1959-1960) \\
(1959-1961) \\
(1959-1960) \\
(1960)\end{array}$ \\
\hline Complessi di Fe: sintesi e struttura & $\begin{array}{l}\text { P. Chini } \\
\text { M. Peraldo }\end{array}$ & $\begin{array}{l}(1960) \\
(1960)\end{array}$ \\
\hline
\end{tabular}

Polimerizzazione isospecifica delle $\alpha$-olefine e polimerizzazione dell'etilene (1954-1970)

\begin{tabular}{|c|c|c|}
\hline Argomento & Collaboratori & Periodo \\
\hline $\begin{array}{l}\text { Sintesi di poli } \alpha \text {-olefine isotattiche } \\
\text { ed a stereoblocchi }\end{array}$ & $\begin{array}{l}\text { P. Pino } \\
\text { G. Mazzanti } \\
\text { U. Giannini } \\
\text { G. Crespi } \\
\text { P. Longi }\end{array}$ & $\begin{array}{r}(1954-1957) \\
(1954-1958) \\
(1957) \\
(1957) \\
(1958)\end{array}$ \\
\hline
\end{tabular}


Segue Polimerizzazione isospecifica delle $\alpha$-olefine e polimerizzazione dell'etilene (1954-1970)

\begin{tabular}{|c|c|c|}
\hline Argomento & Collaboratori & Periodo \\
\hline $\begin{array}{l}\text { Ricerche sui sistemi catalitici e loro } \\
\text { componenti, su complessi organometallici } \\
\text { dell'alluminio e del titanio e meccanismi } \\
\text { di polimerizzazione }\end{array}$ & $\begin{array}{l}\text { P. Pino } \\
\text { G. Mazzanti } \\
\text { U. Giannini } \\
\text { I. Pasquon } \\
\text { E.Giachetti } \\
\text { P. Longi } \\
\text { E. Mantica } \\
\text { F. Danusso } \\
\text { M. Peraldo } \\
\text { P. Corradini } \\
\text { I.W. Bassi } \\
\text { G.F. Pregaglia } \\
\text { M. Farina } \\
\text { D. Morero } \\
\text { S. Cesca } \\
\text { A. Zambelli } \\
\text { M. Bressan } \\
\text { G. Allegra } \\
\text { L. Porri } \\
\text { G. Gatti } \\
\text { P. Ganis } \\
\text { G. Pajaro } \\
\text { A. Immirzi } \\
\text { M. Giongo } \\
\text { A.L. Segre }\end{array}$ & $\begin{array}{r}(1954-1959) \\
(1954-1960) \\
(1957-1967) \\
(1957-1967) \\
(1957-1960) \\
(1957) \\
(1957) \\
(1956-1957) \\
(1957) \\
(1958-1962) \\
(1958) \\
(1959) \\
(1959-1960) \\
(1959-1960) \\
(1959-1960) \\
(1960-1968) \\
(1960) \\
(1960-1963) \\
(1960-1962) \\
(1961-1966) \\
(1961-1962) \\
(1961) \\
(1963) \\
(1968) \\
(1968)\end{array}$ \\
\hline Struttura cristallografica dei polimeri & $\begin{array}{l}\text { P. Corradini } \\
\text { I.W. Bassi } \\
\text { I. Pasquon } \\
\text { M. Cesari } \\
\text { G. Allegra }\end{array}$ & $\begin{array}{r}(1954-1960) \\
(1955-1969) \\
(1955) \\
(1956-1957) \\
(1969)\end{array}$ \\
\hline Polimerizzazione dell'etilene & $\begin{array}{l}\text { P. Pino } \\
\text { M. Farina } \\
\text { G. Mazzanti } \\
\text { G. Giannini } \\
\text { E. Mantica } \\
\text { M. Ragazzini }\end{array}$ & $\begin{array}{r}(1954-1958) \\
(1955-1958) \\
(1957-1958) \\
(1957-1958) \\
(1957) \\
(1958)\end{array}$ \\
\hline
\end{tabular}


Segue Polimerizzazione isospecifica delle $\alpha$-olefine e polimerizzazione dell'etilene (1954-1970)

\begin{tabular}{|c|c|c|}
\hline Argomento & Collaboratori & Periodo \\
\hline $\begin{array}{l}\text { Proprietà fisiche e chimico-fisiche, } \\
\text { determinazione dei pesi molecolari, } \\
\text { conformazione e morfologia dei polimeri }\end{array}$ & $\begin{array}{l}\text { F. Danusso } \\
\text { G. Moraglio } \\
\text { G. Talamini } \\
\text { M. Pegoraro } \\
\text { F. Sabbioni } \\
\text { M. Peraldo } \\
\text { V. Giannella } \\
\text { E. Flores } \\
\text { G. Gianotti } \\
\text { P. Corradini } \\
\text { P. Ganis } \\
\text { G. Polizzotti } \\
\text { G. Allegra }\end{array}$ & $\begin{array}{r}(1954-1969) \\
(1955-1969) \\
(1956-1959) \\
(1958-1964) \\
(1958) \\
(1958) \\
(1958) \\
(1958) \\
(1959-1969) \\
(1962) \\
(1962) \\
(1963-1964) \\
(1970)\end{array}$ \\
\hline $\begin{array}{l}\text { Indagini spettroscopiche sui polimeri: } \\
\text { - Infrarosso }\end{array}$ & $\begin{array}{l}\text { E. Mantica } \\
\text { M. Peraldo } \\
\text { G. Zerbi } \\
\text { F. Ciampelli } \\
\text { V. Zamboni } \\
\text { M. Gussoni }\end{array}$ & $\begin{array}{r}(1955) \\
(1955-1959) \\
(1964-1968) \\
(1964-1967) \\
(1967) \\
(1967)\end{array}$ \\
\hline $\begin{array}{l}\text { - Risonanza magnetica nucleare } \\
\text { - Raman-Laser }\end{array}$ & $\begin{array}{l}\text { E. Lombardi } \\
\text { A. Zambelli } \\
\text { A.L. Segre } \\
\text { L. Piseri } \\
\text { G. Zerbi }\end{array}$ & $\begin{array}{r}(1965-1967) \\
(1965-1970) \\
(1965-1968) \\
(1968) \\
(1969)\end{array}$ \\
\hline $\begin{array}{l}\text { Cinetica della polimerizzazione; } \\
\text { stadi elementari; regolazione e distribuzione } \\
\text { dei pesi molecolari }\end{array}$ & $\begin{array}{l}\text { I. Pasquon } \\
\text { E. Giachetti } \\
\text { L. Giuffrè } \\
\text { G. Pajaro } \\
\text { M. Dente } \\
\text { G. Mazzanti } \\
\text { P. Longi } \\
\text { F. Bernardini } \\
\text { A. Zambelli } \\
\text { M. Giongo } \\
\text { A. Marinangeli } \\
\text { A. Surico }\end{array}$ & $\begin{array}{r}(1956-1967) \\
(1956-1960) \\
(1958-1961) \\
(1958) \\
(1959-1962) \\
(1959) \\
(1959) \\
(1959) \\
(1962-1967) \\
(1966) \\
(1967) \\
(1967)\end{array}$ \\
\hline $\begin{array}{l}\text { Proprietà meccaniche e tecnologiche } \\
\text { dei polimeri }\end{array}$ & $\begin{array}{l}\text { G. Crespi } \\
\text { M. Baccaredda } \\
\text { E. Butta } \\
\text { F. Ranalli }\end{array}$ & $\begin{array}{r}(1957-1959) \\
(1959) \\
(1959) \\
(1959)\end{array}$ \\
\hline
\end{tabular}


Segue Polimerizzazione isospecifica delle $\alpha$-olefine e polimerizzazione dell'etilene (1954-1970)

\begin{tabular}{|c|c|c|}
\hline Argomento & Collaboratori & Periodo \\
\hline $\begin{array}{l}\text { Determinazione analitiche su polimeri } \\
\text { e catalizzatori }\end{array}$ & $\begin{array}{l}\text { L. Giuffrè } \\
\text { F.M. Capizzi } \\
\text { F. Cassani } \\
\text { E. Losio }\end{array}$ & $\begin{array}{l}(1958-1967) \\
(1959-1962) \\
(1964-1966) \\
(1965-1967)\end{array}$ \\
\hline
\end{tabular}

Polimerizzazione isospecifica e copolimerizzazione dello stirene e di altri monomeri vinilaromatici diversamente sostituiti (1954-1969)

\begin{tabular}{|c|c|c|}
\hline Argomento & Collaboratori & Periodo \\
\hline $\begin{array}{l}\text { Sintesi dei polimeri e dei copolimeri, } \\
\text { sistemi catalitici; cinetica; } \\
\text { meccanismi di polimerizzazione }\end{array}$ & $\begin{array}{l}\text { F. Danusso } \\
\text { D. Sianesi } \\
\text { B. Calcagno } \\
\text { A. Macchi } \\
\text { G. Dall'Asta } \\
\text { P. Ferruti } \\
\text { G. Moraglio } \\
\text { A. Feré }\end{array}$ & $\begin{array}{r}(1954-1965) \\
(1956-1962) \\
(1958-1959) \\
(1959) \\
(1962) \\
(1965) \\
(1968) \\
(1968)\end{array}$ \\
\hline Struttura cristallografica dei polimeri & $\begin{array}{l}\text { P. Corradini } \\
\text { I.W. Bassi } \\
\text { P. Ganis }\end{array}$ & $\begin{array}{r}(1955-1960) \\
(1960) \\
(1960)\end{array}$ \\
\hline $\begin{array}{l}\text { Cristallizzabilità, morfologia cristallina, } \\
\text { conformazioni statistiche, proprietà fisiche e } \\
\text { chimico-fisiche dei polimeri }\end{array}$ & $\begin{array}{l}\text { F. Danusso } \\
\text { G. Moraglio } \\
\text { F. Sabbioni }\end{array}$ & $\begin{array}{r}(1955-1963) \\
(1956-1965) \\
(1958)\end{array}$ \\
\hline Indagini spettroscopiche sui polimeri: & & \\
\hline $\begin{array}{l}\text { - Infrarosso } \\
\text { - Risonanza magnetica nucleare }\end{array}$ & $\begin{array}{l}\text { D. Morero } \\
\text { E. Mantica } \\
\text { F. Ciampelli } \\
\text { A.L. Segre } \\
\text { P. Ferruti }\end{array}$ & $\begin{array}{l}(1960) \\
(1960) \\
(1960) \\
(1969) \\
(1969)\end{array}$ \\
\hline
\end{tabular}


Polimerizzazione e copolimerizzazione di diolefine coniugate. Polimeri otticamente attivi (1955-1970)

\begin{tabular}{|c|c|c|}
\hline Argomento & Collaboratori & Periodo \\
\hline $\begin{array}{l}\text { Sintesi di polibutadieni stereoregolari } \\
(1,2 \text { iso- e } 1,2 \text { sindiotattico, 1,4-cis e 1,4-trans })\end{array}$ & L. Porri & $(1955-1957)$ \\
\hline $\begin{array}{l}\text { Sintesi di polisopreni stereoregolari } \\
(1,4 \text {-cis, 1,4-trans e } 3,4)\end{array}$ & $\begin{array}{l}\text { L. Porri } \\
\text { A. Carbonaro }\end{array}$ & $\begin{array}{r}(1956-1964) \\
(1964)\end{array}$ \\
\hline $\begin{array}{l}\text { Sintesi di polipentadieni } 1,3 \text { stereoregolari } \\
\text { (1,4-trans isotattico, } 1,4 \text {-cis sindio- } \\
\text { e isotattici; } 1,2 \text { sindiotattico) } \\
\text { e di altri polibutadieni sostituiti }\end{array}$ & $\begin{array}{l}\text { L. Porri } \\
\text { G. Zanini } \\
\text { A. Carbonaro } \\
\text { M.C. Gallazzi }\end{array}$ & $\begin{array}{r}(1960-1966) \\
(1960) \\
(1962) \\
(1966)\end{array}$ \\
\hline $\begin{array}{l}\text { Sintesi di polipentadieni 1,4-trans isotattico } \\
\text { e 1,4-cis otticamente attivi }\end{array}$ & $\begin{array}{l}\text { L. Porri } \\
\text { A. Carbonaro } \\
\text { G. Lugli } \\
\text { S. Valenti }\end{array}$ & $\begin{array}{r}(1961-1963) \\
(1961-1963) \\
(1961-1963) \\
(1963)\end{array}$ \\
\hline Struttura di polidiolefine coniugate & $\begin{array}{l}\text { P. Corradini } \\
\text { I.W. Bassi } \\
\text { G. Allegra } \\
\text { G. Perego } \\
\text { P. Ganis }\end{array}$ & $\begin{array}{r}(1955-1968) \\
(1957-1968) \\
(1962-1963) \\
(1963-1964) \\
(1965)\end{array}$ \\
\hline $\begin{array}{l}\text { Ricerche sulla polimerizzazione } \\
\text { stereospecifica delle diolefine coniugate: } \\
\text { sistemi catalitici, meccanismi } \\
\text { di polimerizzazione; complessi organometallici } \\
\text { con diolefine }\end{array}$ & $\begin{array}{l}\text { L. Porri } \\
\text { G. Zanini } \\
\text { A. Mazzei } \\
\text { L. Fiore } \\
\text { A. Palvarini } \\
\text { A. Carbonaro } \\
\text { L. Borghi } \\
\text { G. Lugli } \\
\text { S. Valenti } \\
\text { A. Greco } \\
\text { M.C. Gallazzi } \\
\text { G. Allegra } \\
\text { U. Giannini } \\
\text { P. Pino } \\
\text { G. Sovarzi } \\
\text { A. Lionetti } \\
\text { M. Donati } \\
\text { F. Conti } \\
\text { G. Vitulli } \\
\text { A. Di Corato } \\
\text { M. Zocchi }\end{array}$ & $\begin{array}{r}(1956-1970) \\
(1959-1961) \\
(1959) \\
(1959) \\
(1959) \\
(1960-1968) \\
(1960) \\
(1961-1963) \\
(1963) \\
(1964-1968) \\
(1964-1967) \\
(1965-1969) \\
(1965) \\
(1965) \\
(1965) \\
(1965-1966) \\
(1966) \\
(1966) \\
(1967-1969) \\
(1967-1969) \\
(1969)\end{array}$ \\
\hline
\end{tabular}


Segue Polimerizzazione e copolimerizzazione di diolefine coniugate. Polimeri otticamente attivi (1955-1970)

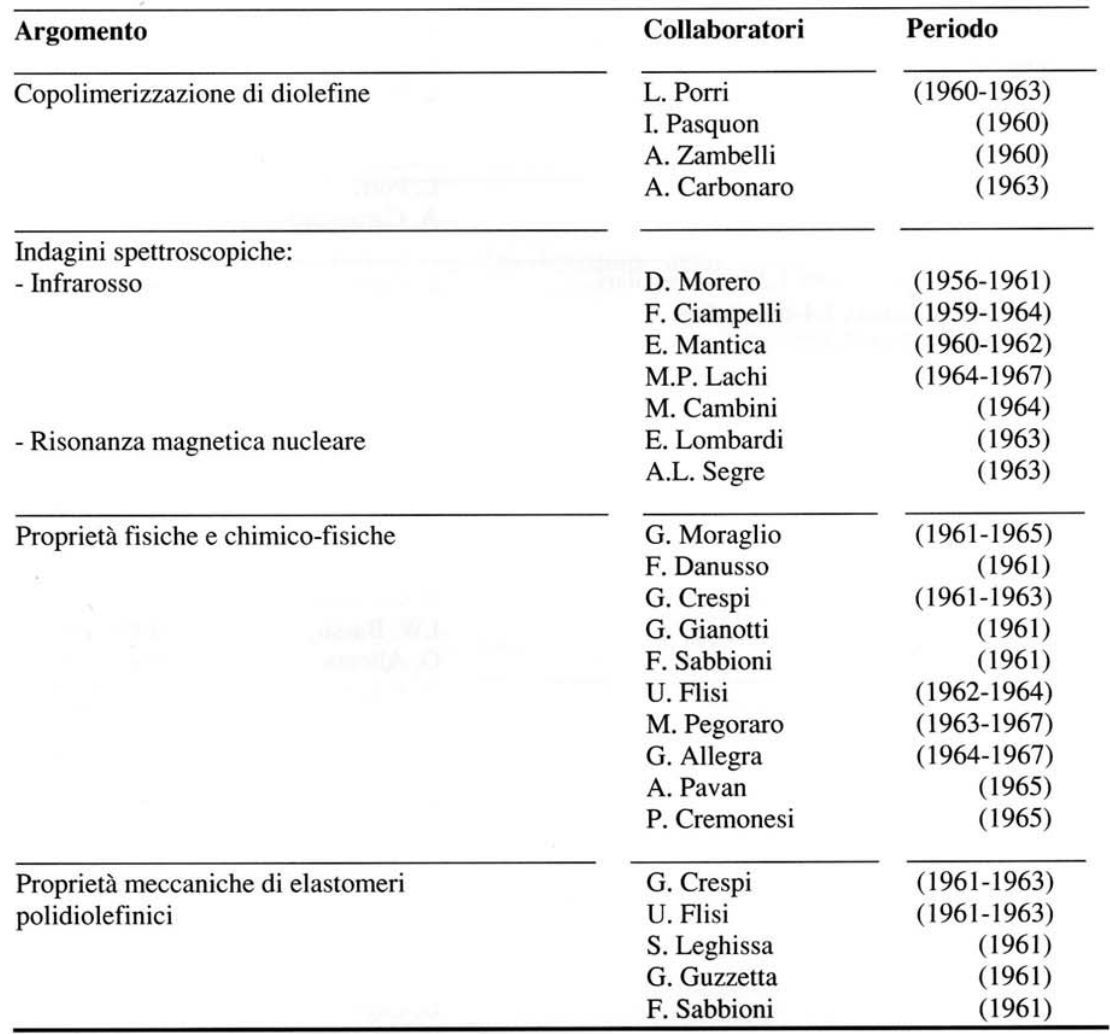


Copolimerizzazione etilene-propilene ed altre $\boldsymbol{\alpha}$-olefine. Elastomeri poliolefinici (1957-1967)

\begin{tabular}{|c|c|c|}
\hline Argomento & Collaboratori & Periodo \\
\hline Sintesi dei copolimeri & $\begin{array}{l}\text { G. Mazzanti } \\
\text { A. Valvassori } \\
\text { G. Sartori } \\
\text { I. Pasquon } \\
\text { A. Zambelli } \\
\text { L. Porri } \\
\text { F. Ciampelli } \\
\text { S. Faina }\end{array}$ & $\begin{array}{r}(1957-1961) \\
(1957-1965) \\
(1960-1965) \\
(1961-1963) \\
(1961-1963) \\
(1961) \\
(1962) \\
(1965)\end{array}$ \\
\hline $\begin{array}{l}\text { Studio della copolimerizzazione: rapporti } \\
\text { di reattività; cinetica; pesi molecolari; } \\
\text { distribuzione delle unità monomeriche }\end{array}$ & $\begin{array}{l}\text { A. Valvassori } \\
\text { F. Mazzanti } \\
\text { G. Sartori } \\
\text { G. Pajaro } \\
\text { G. Moraglio } \\
\text { D. Morero } \\
\text { F. Ciampelli }\end{array}$ & $\begin{array}{r}(1957-1967) \\
(1957-1963) \\
(1958-1963) \\
(1957-1963) \\
(1959) \\
(1960) \\
(1967)\end{array}$ \\
\hline $\begin{array}{l}\text { Preparazione, proprietà fisiche e meccaniche, } \\
\text { vulcanizzazione degli elastomeri poliolefinici }\end{array}$ & $\begin{array}{l}\text { G. Crespi } \\
\text { M. Bruzzone } \\
\text { E. Di Giulio } \\
\text { P. Scaglione } \\
\text { G. Ballini } \\
\text { G. Flisi } \\
\text { A. Valvassori } \\
\text { G. Mazzanti } \\
\text { G. Sartori }\end{array}$ & $\begin{array}{r}(1959-1964) \\
(1959-1961) \\
(1961-1963) \\
(1961) \\
(1961) \\
(1963-1964) \\
(1963) \\
(1963) \\
(1963)\end{array}$ \\
\hline
\end{tabular}


Polimerizzazione sterospecifica di $\alpha$-olefine diversamente sostituite (1955-1968)

\begin{tabular}{|c|c|c|}
\hline Argomento & Collaboratori & Periodo \\
\hline \multicolumn{3}{|l|}{ Sintesi dei polimeri isotattici e sistemi catalitici: } \\
\hline \multirow{4}{*}{$\begin{array}{l}\text { - Polimeri di } \alpha \text {-olefine sostituite con metalli } \\
\text { del IV gruppo }\end{array}$} & G. Mazzanti & $(1958-1959)$ \\
\hline & P. Longi & $(1958-1959)$ \\
\hline & F. Bernardini & $(1958-1959)$ \\
\hline & G. Giannini & (1968) \\
\hline - Polivinilcicloesano & D. Sianesi & (1959) \\
\hline \multirow{5}{*}{ - Polivinilisobutileteri } & G. Dall'Asta & $(1959-1963)$ \\
\hline & G. Mazzanti & (1959) \\
\hline & G. Giannini & (1959) \\
\hline & S. Cesca & (1959) \\
\hline & N. Oddo & (1960) \\
\hline \multirow[t]{2}{*}{ - Polivinilciclopropano } & D. Sianesi & $(1960-1961)$ \\
\hline & G. Caporiccio & $(1960)$ \\
\hline \multirow[t]{2}{*}{ - Poli $\alpha$-olefine con gruppi fenilici terminali } & G.F. Pregaglia & $(1960-1961)$ \\
\hline & M. Binaghi & $(1960)$ \\
\hline \multirow{4}{*}{ - Poli 2-vinilpiridina } & G. Mazzanti & $(1960-1961)$ \\
\hline & P. Longi & $(1960-1961)$ \\
\hline & G. Dall'Asta & $(1961)$ \\
\hline & F. Bernardini & (1961) \\
\hline \multirow{3}{*}{ - Poliacrilati e polimetacrilati } & G. Mazzanti & (1960) \\
\hline & P. Longi & (1960) \\
\hline & F. Bernardini & (1960) \\
\hline - Poli N-vinildifenilammina & P. Longi & (1961) \\
\hline - Poli $\alpha$-alchilacrilonitrile & G. Dall'Asta & (1964) \\
\hline - Poli N-vinilcarbazolo & G. Dall'Asta & (1965) \\
\hline \multirow{2}{*}{ - Poli viniletilsilano } & A. Carbonaro & (1968) \\
\hline & A. Greco & (1968) \\
\hline \multirow[t]{2}{*}{ Sintesi del polivinilcloruro sindiotattico } & U. Giannini & (1962) \\
\hline & S. Cesca & (1962) \\
\hline \multirow[t]{3}{*}{ Struttura dei polimeri } & I.W. Bassi & $(1955-1968)$ \\
\hline & P. Corradini & $(1955-1959)$ \\
\hline & G. Allegra & $(1965)$ \\
\hline \multirow[t]{2}{*}{ Indagini spettroscopiche } & M. Peraldo & $(1960-1961)$ \\
\hline & F. Ciampelli & $(1966)$ \\
\hline \multirow{2}{*}{$\begin{array}{l}\text { Proprietà fisiche e chimico-fisiche } \\
\text { del PVC cristallino }\end{array}$} & F. Danusso & (1968) \\
\hline & A. Crugnola & (1968) \\
\hline
\end{tabular}


Polimeri vari: sintesi, struttura, caratterizzazione, sistemi catalitici (1956-1970)

\begin{tabular}{|c|c|c|}
\hline Argomento & Collaboratori & Periodo \\
\hline Polipropilenossido cristallino & $\begin{array}{l}\text { G. Dall'Asta } \\
\text { P. Corradini }\end{array}$ & $\begin{array}{l}(1956) \\
(1956)\end{array}$ \\
\hline Poliacetilene & $\begin{array}{l}\text { G. Mazzanti } \\
\text { P. Corradini } \\
\text { E. Lombardi } \\
\text { L: Giuffrè } \\
\text { L. Porri } \\
\text { M.C. Gallazzi } \\
\text { G. Vitulli }\end{array}$ & $\begin{array}{l}(1958) \\
(1958) \\
(1958) \\
(1958) \\
(1967) \\
(1967) \\
(1967)\end{array}$ \\
\hline Poliesino-1 & $\begin{array}{l}\text { G. Mazzanti } \\
\text { G.F. Pregaglia } \\
\text { M. Peraldo }\end{array}$ & $\begin{array}{l}(1959) \\
(1959) \\
(1959)\end{array}$ \\
\hline Polialdeidi superiori isotattiche & $\begin{array}{l}\text { G. Mazzanti } \\
\text { P. Chini } \\
\text { A. Valvassori } \\
\text { P. Corradini } \\
\text { I.W. Bassi }\end{array}$ & $\begin{array}{r}(1960) \\
(1960) \\
(1960) \\
(1960-1961) \\
(1960-1961)\end{array}$ \\
\hline Poliallilbenzene & $\begin{array}{l}\text { G.F. Pregaglia } \\
\text { M. Binaghi }\end{array}$ & $\begin{array}{l}(1961) \\
(1961)\end{array}$ \\
\hline Poliisocianati cristallini & $\begin{array}{l}\text { J. Di Pietro } \\
\text { M. Cambini }\end{array}$ & $\begin{array}{l}(1962) \\
(1962)\end{array}$ \\
\hline Poliallilacrilati & $\begin{array}{l}\text { M. Donati } \\
\text { M. Farina }\end{array}$ & $\begin{array}{l}(1963) \\
(1963)\end{array}$ \\
\hline Poli 1-cianobutadiene 1,3 & $\begin{array}{l}\text { G. Giannini } \\
\text { M. Cambini } \\
\text { A. Cassata }\end{array}$ & $\begin{array}{l}(1963) \\
(1963) \\
(1963)\end{array}$ \\
\hline Poli trans 1,2-divinilciclobutano & $\begin{array}{l}\text { A. Valvassori } \\
\text { G. Sartori } \\
\text { F. Ciampelli }\end{array}$ & $\begin{array}{l}(1963) \\
(1963) \\
(1963)\end{array}$ \\
\hline Polinorborneni & $\begin{array}{l}\text { G. Sartori } \\
\text { A.Valvassori } \\
\text { N. Canali } \\
\text { F. Ciampelli }\end{array}$ & $\begin{array}{r}(1963-1964) \\
(1964) \\
(1963) \\
(1963)\end{array}$ \\
\hline
\end{tabular}


Segue Polimeri vari: sintesi, struttura, caratterizzazione, sistemi catalitici (1956-1970)

\begin{tabular}{|c|c|c|}
\hline Argomento & Collaboratori & Periodo \\
\hline Copolimeri della formaldeide & $\begin{array}{l}\text { G.F. Pregaglia } \\
\text { G. Mazzanti } \\
\text { M. Binaghi } \\
\text { G. Rebaudo } \\
\text { V. Zamboni }\end{array}$ & $\begin{array}{l}(1963) \\
(1963) \\
(1963) \\
(1968) \\
(1968)\end{array}$ \\
\hline Poli $\beta$-(N-carbonil) etilmetacrilato & $\begin{array}{l}\text { P. Longi } \\
\text { E. Pellini }\end{array}$ & $\begin{array}{l}(1964) \\
(1964)\end{array}$ \\
\hline Polilattoni e policiclochetoni & $\begin{array}{l}\text { P. Longi } \\
\text { F. Greco } \\
\text { M. Mapelli }\end{array}$ & $\begin{array}{l}(1965) \\
(1965) \\
(1965)\end{array}$ \\
\hline Policiclodiolefine & $\begin{array}{l}\text { A. Carbonaro } \\
\text { A. Greco } \\
\text { G. Dall'Asta } \\
\text { A. Valvassori } \\
\text { G. Sartori }\end{array}$ & $\begin{array}{l}(1967) \\
(1967) \\
(1967) \\
(1967) \\
(1967)\end{array}$ \\
\hline Poliallene & $\begin{array}{l}\text { L. Porri } \\
\text { M.C. Gallazzi } \\
\text { G. Vitulli }\end{array}$ & $\begin{array}{l}(1967) \\
(1967) \\
(1967)\end{array}$ \\
\hline Politetraindano e politetraindene & $\begin{array}{l}\text { G. Dall'Asta } \\
\text { G. Motroni }\end{array}$ & $\begin{array}{l}(1968) \\
(1968)\end{array}$ \\
\hline $\begin{array}{l}\text { Oligomeri del norbornadiene con } \\
\text { idrocarburi acetilenici }\end{array}$ & $\begin{array}{l}\text { A. Carbonaro } \\
\text { A. Greco } \\
\text { G. Dall'Asta }\end{array}$ & $\begin{array}{l}(1968) \\
(1968) \\
(1968) \\
\end{array}$ \\
\hline
\end{tabular}

Copolimeri ad innesto: preparazione e studio delle proprietà (1958-1971)

\begin{tabular}{|c|c|c|}
\hline Argomento & Collaboratori & Periodo \\
\hline $\begin{array}{l}\text { Poli } \alpha \text {-olefine innestate con polistirene, } \\
\text { poliacrilato, polimetacrilato di metile } \\
\text { o poliacido acrilico }\end{array}$ & $\begin{array}{l}\text { E. Beati } \\
\text { M. Pegoraro } \\
\text { F. Severini } \\
\text { D. Pagani } \\
\text { C. Tavazzani } \\
\text { A. Penati }\end{array}$ & $\begin{array}{r}(1958-1968) \\
(1960-1968) \\
(1958-1968) \\
(1964) \\
(1968) \\
(1969)\end{array}$ \\
\hline
\end{tabular}


Segue Copolimeri ad innesto: preparazione e studio delle proprietà (1958-1971)

\begin{tabular}{|c|c|c|}
\hline Argomento & Collaboratori & Periodo \\
\hline $\begin{array}{l}\text { Copolimeri etilene-propilene innestati } \\
\text { con polistirene, policloruro di vinile, } \\
\text { poliacido acrilico o poliacetato di vinile }\end{array}$ & $\begin{array}{l}\text { M. Pegoraro } \\
\text { F. Severini } \\
\text { A. Crugnola } \\
\text { C. Tavazzani } \\
\text { F. Danusso }\end{array}$ & $\begin{array}{r}(1965-1971) \\
(1965-1970) \\
(1965-1969) \\
(1967) \\
(1969)\end{array}$ \\
\hline Analisi di polimeri perossidati & L. Giuffrè & $(1964-1968)$ \\
\hline
\end{tabular}

Polimeri politattici. Sintesi asimmetrica (1958-1969)

\begin{tabular}{|c|c|c|}
\hline Argomento & Collaboratori & Periodo \\
\hline Polimeri diisotattici & $\begin{array}{l}\text { M. Farina } \\
\text { M. Peraldo } \\
\text { G. Bressan }\end{array}$ & $\begin{array}{r}(1958-1962) \\
(1958-1962) \\
(1962)\end{array}$ \\
\hline Polimeri tritattici & $\begin{array}{l}\text { M. Farina } \\
\text { M. Donati } \\
\text { M. Peraldo }\end{array}$ & $\begin{array}{r}(1960-1965) \\
(1960-1965) \\
(1960)\end{array}$ \\
\hline Sintesi asimmetrica di polimeri politattici & $\begin{array}{l}\text { M. Farina } \\
\text { M. Donati } \\
\text { M. Peraldo } \\
\text { M. Bressan }\end{array}$ & $\begin{array}{r}(1960-1969) \\
(1960) \\
(1961) \\
(1961-1963)\end{array}$ \\
\hline $\begin{array}{l}\text { Sintesi asimmetrica di polibenzo-e } \\
\text { polinaftofurani }\end{array}$ & $\begin{array}{l}\text { M. Farina } \\
\text { G. Bressan }\end{array}$ & $\begin{array}{l}(1963-1966) \\
(1963-1966)\end{array}$ \\
\hline Struttura dei polimeri cristallini & $\begin{array}{l}\text { P. Corradini } \\
\text { P. Ganis }\end{array}$ & $\begin{array}{r}(1960-1963) \\
(1963)\end{array}$ \\
\hline
\end{tabular}

Polimerizzazione sindiospecifica del propilene (1960-1969)

\begin{tabular}{|c|c|c|}
\hline Argomento & Collaboratori & Periodo \\
\hline Sintesi del polimero & $\begin{array}{l}\text { I. Pasquon } \\
\text { A. Zambelli }\end{array}$ & $\begin{array}{l}(1960) \\
(1960)\end{array}$ \\
\hline Struttura e conformazione del polimero & $\begin{array}{l}\text { P. Corradini } \\
\text { M. Peraldo } \\
\text { G. Allegra } \\
\text { A. Zambelli } \\
\text { G. Zerbi }\end{array}$ & $\begin{array}{r}(1960) \\
(1960-1965) \\
(1964) \\
(1965) \\
(1969)\end{array}$ \\
\hline
\end{tabular}


Segue Polimerizzazione sindiospecifica del propilene (1960-1969)

\begin{tabular}{|c|c|c|}
\hline Argomento & Collaboratori & Periodo \\
\hline $\begin{array}{l}\text { Ricerche sui sistemi catalitici; cinetica; } \\
\text { meccanismi di polimerizzazione }\end{array}$ & $\begin{array}{l}\text { A. Zambelli } \\
\text { I. Pasquon } \\
\text { S. Signorini } \\
\text { M. Giongo } \\
\text { G. Tosi }\end{array}$ & $\begin{array}{r}(1961-1968) \\
(1961-1968) \\
(1967-1968) \\
(1968) \\
(1968)\end{array}$ \\
\hline Separazione e proprietà fisiche del polimero & M. Pegoraro & $(1960-1963)$ \\
\hline
\end{tabular}

Copolimeri alternanti cristallini dell'etilene e della 2-vinilpiridina (1961-1965)

\begin{tabular}{|c|c|c|}
\hline Argomento & Collaboratori & Periodo \\
\hline $\begin{array}{l}\text { Copolimeri dell'etilene con butene-2, } \\
\text { cicloolefine o butadiene: sintesi e } \\
\text { sistemi catalitici }\end{array}$ & $\begin{array}{l}\text { G. Dall'Asta } \\
\text { I. Pasquon } \\
\text { A. Zambelli } \\
\text { A. Valvassori } \\
\text { G. Mazzanti }\end{array}$ & $\begin{array}{l}(1961-1962) \\
(1961-1964) \\
(1961-1964) \\
(1961-1962) \\
(1961-1962)\end{array}$ \\
\hline Struttura dei copolimeri & $\begin{array}{l}\text { P. Corradini } \\
\text { I.W. Bassi } \\
\text { P. Ganis } \\
\text { G. Allegra } \\
\text { F. Ciampelli }\end{array}$ & $\begin{array}{r}(1962-1964) \\
(1962-1964) \\
(1962-1964) \\
(1962-1964) \\
(1964)\end{array}$ \\
\hline $\begin{array}{l}\text { Copolimeri 2-vinilpiridina-stilbazolo: } \\
\text { sintesi e sistemi catalitici }\end{array}$ & $\begin{array}{l}\text { P. Longi } \\
\text { U. Nordio }\end{array}$ & $\begin{array}{l}(1965) \\
(1965)\end{array}$ \\
\hline
\end{tabular}

Policicloolefine stereoregolari. Elastomeri (1962-1968)

\begin{tabular}{|c|c|c|}
\hline Argomento & Collaboratori & Periodo \\
\hline $\begin{array}{l}\text { Policiclobutenameri: sintesi, sistemi catalitici } \\
\text { e meccanismi }\end{array}$ & $\begin{array}{l}\text { G. Dall'Asta } \\
\text { G. Motroni } \\
\text { L. Porri } \\
\text { G. Mazzanti } \\
\text { R. Manetti } \\
\text { G. Carella }\end{array}$ & $\begin{array}{r}(1962-1968) \\
(1963-1968) \\
(1962-1965) \\
(1962-1964) \\
(1966-1968) \\
(1966)\end{array}$ \\
\hline $\begin{array}{l}\text { Policicloolefine superiori: sintesi, } \\
\text { sistemi catalitici e meccanismi }\end{array}$ & $\begin{array}{l}\text { G. Dall'Asta } \\
\text { G. Mazzanti } \\
\text { L. Porri } \\
\text { M.C. Gallazzi } \\
\text { G. Carella }\end{array}$ & $\begin{array}{r}(1964-1966) \\
(1964) \\
(1964) \\
(1964) \\
(1966)\end{array}$ \\
\hline
\end{tabular}


Segue Policicloolefine stereoregolari. Elastomeri (1962-1968)

\begin{tabular}{|c|c|c|}
\hline Argomento & Collaboratori & Periodo \\
\hline Struttura dei polimeri & I.W. Bassi & (1966) \\
\hline Elastomeri da cicloolefine & G. Dall'Asta & (1968) \\
\hline
\end{tabular}

Polimeri e copolimeri stereoregolari del chetene e del dimetilchetene: sintesi, caratterizzazione e sistemi catalitici (1960-1963)

\begin{tabular}{|c|c|c|}
\hline Argomento & Collaboratori & Periodo \\
\hline Polimeri del chetene & $\begin{array}{l}\text { G.F. Pregaglia } \\
\text { G. Pozzi }\end{array}$ & $\begin{array}{l}(1963) \\
(1963)\end{array}$ \\
\hline Polimeri del dimetilchetene & $\begin{array}{l}\text { G. Mazzanti } \\
\text { G.F. Pregaglia } \\
\text { M. Binaghi } \\
\text { M. Cambini }\end{array}$ & $\begin{array}{r}(1960-1962) \\
(1960-1962) \\
(1960-1962) \\
(1962)\end{array}$ \\
\hline $\begin{array}{l}\text { Copolimeri alternanti del dimetilchetene } \\
\text { con acetone o aldeidi }\end{array}$ & $\begin{array}{l}\text { G.F. Pregaglia } \\
\text { M. Binaghi } \\
\text { G. Mazzanti } \\
\text { G. Pozzi } \\
\text { V. Zamboni }\end{array}$ & $\begin{array}{r}(1960-1963) \\
(1960-1963) \\
(1960-1962) \\
(1962) \\
(1963)\end{array}$ \\
\hline
\end{tabular}

Poliolefine fluorurate (1961-1970)

\begin{tabular}{|c|c|c|}
\hline Argomento & Collaboratori & Periodo \\
\hline Sintesi, sistemi catalitici & $\begin{array}{l}\text { D. Sianesi } \\
\text { G. Caporiccio }\end{array}$ & $\begin{array}{l}(1963-1965) \\
(1963-1965)\end{array}$ \\
\hline Struttura dei polimeri & $\begin{array}{l}\text { I.W. Bassi } \\
\text { G. Allegra }\end{array}$ & $\begin{array}{l}(1961-1965) \\
(1961-1965)\end{array}$ \\
\hline Indagini all'infrarosso & $\begin{array}{l}\text { G. Cortili } \\
\text { G. Zerbi }\end{array}$ & $\begin{array}{l}(1967-1970) \\
(1967-1970)\end{array}$ \\
\hline
\end{tabular}


Composti di inclusione - Polimerizzazione di monomeri inclusi - Polimerizzazione asimmetrica (1964-1970)

\begin{tabular}{|c|c|c|}
\hline Argomento & Collaboratori & Periodo \\
\hline $\begin{array}{l}\text { Composti di inclusione in peridrotrifenilene } \\
\text { e polimerizzazione radicalica di diolefine } \\
\text { incluse }\end{array}$ & $\begin{array}{l}\text { M. Farina } \\
\text { G. Audisio } \\
\text { M. Löffelholz }\end{array}$ & $\begin{array}{l}(1964-1970) \\
(1967-1970) \\
(1967-1968)\end{array}$ \\
\hline $\begin{array}{l}\text { Polimerizzazione asimmetrica di trans-1,3 } \\
\text { pentadiene incluso }\end{array}$ & $\begin{array}{l}\text { M. Farina } \\
\text { G. Audisio } \\
\text { M.T. Gramegna }\end{array}$ & $\begin{array}{r}(1967-1970) \\
(1967-1970) \\
(1970)\end{array}$ \\
\hline Indagini roentgenografiche & $\begin{array}{l}\text { G. Allegra } \\
\text { A. Immirzi } \\
\text { A. Colombo }\end{array}$ & $\begin{array}{r}(1964-1968) \\
(1967) \\
(1967)\end{array}$ \\
\hline
\end{tabular}

Polimeri per applicazioni biomediche e farmacologiche (1965-1970)

\begin{tabular}{|c|c|c|}
\hline Argomento & Collaboratori & Periodo \\
\hline Polimeri per la prevenzione della silicosi & $\begin{array}{l}\text { F. Danusso } \\
\text { P. Ferruti } \\
\text { M.A. Marchisio } \\
\text { E. Vigliani } \\
\text { E. Pernis }\end{array}$ & $\begin{array}{l}(1965-1966) \\
(1965-1966) \\
(1965-1968) \\
(1965-1966) \\
(1965-1966)\end{array}$ \\
\hline Polimeri con funzioni amminiche & $\begin{array}{l}\text { P. Ferruti } \\
\text { F. Danusso }\end{array}$ & $\begin{array}{l}(1966-1970) \\
(1966-1970)\end{array}$ \\
\hline $\begin{array}{l}\text { Polimeri con funzioni amminiche, ammidiche } \\
\text { e amminiche. Materiali emocompatibili } \\
\text { e antagonisti dell'eparina }\end{array}$ & $\begin{array}{l}\text { F. Danusso } \\
\text { P. Ferruti } \\
\text { F. Ferroni } \\
\text { M.A. Marchisio }\end{array}$ & $\begin{array}{r}(1967-1970) \\
(1967-1970) \\
(1967) \\
(1970)\end{array}$ \\
\hline
\end{tabular}

